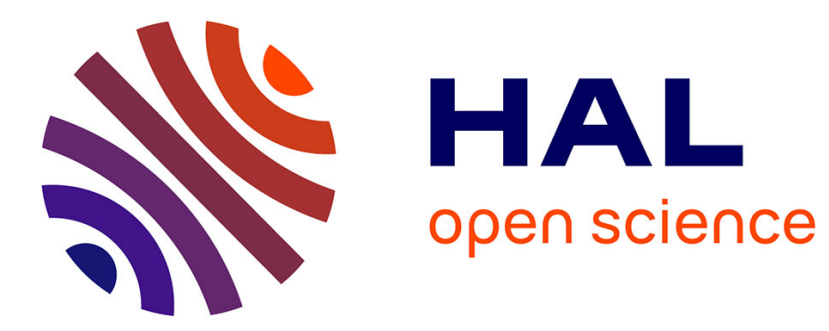

\title{
Invariant measures for some partial differential equations
} Brigitte Bidégaray

\section{To cite this version:}

Brigitte Bidégaray. Invariant measures for some partial differential equations. Physica D: Nonlinear Phenomena, 1995, 82 (4), pp.340-364. 10.1016/0167-2789(94)00238-L . hal-00319984

\section{HAL Id: hal-00319984 \\ https://hal.science/hal-00319984}

Submitted on 9 Jun 2021

HAL is a multi-disciplinary open access archive for the deposit and dissemination of scientific research documents, whether they are published or not. The documents may come from teaching and research institutions in France or abroad, or from public or private research centers.
L'archive ouverte pluridisciplinaire HAL, est destinée au dépôt et à la diffusion de documents scientifiques de niveau recherche, publiés ou non, émanant des établissements d'enseignement et de recherche français ou étrangers, des laboratoires publics ou privés. 


\title{
Invariant measures for some partial differential equations
}

\author{
B. Bidégaray
}

\author{
Centre de Mathématiques et de Leurs Applications \\ ENS de Cachan et URA 1611 \\ 61 avenue du président Wilson \\ 94235 Cachan, France \\ and \\ Laboratoire d'Analyse Numérique, Bât. 425 \\ Université de Paris-Sud et CNRS \\ 91405 Orsay, France
}

\begin{abstract}
We construct invariant measures for Hamiltonian systems such as the nonlinear Schrödinger equation or the wave equation in order to prove Poisson's recurrence. The particular case of schemes (finite dimensional spaces) is also treated in order to explain the recurrence phenomenon which is observed during numerical simulations.
\end{abstract}

AMS Classification : 28C20, 35B15, 35Q20 


\section{Introduction.}

Fermi-Pasta-Ulam recurrence phenomenon has first been noticed in the context of the numerical study of a chain of balls with nonlinear interactions. This phenomenon has also been observed experimentally by Yuen, Lake and Ferguson [23] in the frame of deep water waves governed by the cubic nonlinear Schrödinger equation. This phenomenon may be described as follows. The energy of an initial data with a finite number of modes spreads to higher modes and after a certain lapse of time we observe a return to the initial modes. Such a behavior is "almost" periodic in time.

The propagation of energy to higher modes is connected with an other phenomenon which also occurs when dealing with certain equations with periodic boundary conditions : Benjamin-Feir instability. This new phenomenon is the instability of spatially uniform solutions for perturbations with a certain frequency.

The link between both effects is made clear in [21] by Yuen and Ferguson. Their numerical results tend to prove that a simple recurrence seems to appear only in the case when the higher modes of the perturbation are stable according to a Benjamin-Feir analysis. These observations are carried out in the 1-dimensional case and generalized by the same authors to the 2-dimensional case in [22]. However, this time, they do not produce the link with BenjaminFeir instability. This link is given in the article [16] by Martin and Yuen. Thanks to a multiple scale method for the time variable, Janssen (cf. [11]) proves the recurrence for certain perturbations of the uniform solution and shows that in such a case the recurrence time is connected with the amplitude in a straightforward way. Using an Ansatz on the form of the solutions (3 Fourier modes) Infeld in [10] shows the recurrence in time of these modes. In [20] Weideman and Herbst use such an approach for this equation but they consider it as schemes and not as Ansatz. Recurrence for the Davey-Stewartson equations has also been studied by K. Rachid using different methods.

In [3], Bourgain shows that the solution to the Korteweg-de Vries equation is almost periodic in time for initial data in $L^{2}$ using the theory of Hill's operator with a periodic $L^{2}$ potential. The same sort of result has been previously obtained by McKean and Trubowitz [15] for initial data in $\mathcal{C}^{\infty}$.

Lax in [12] constructs some particular solutions to the Korteweg-de Vries equation verifying a minimization problem with constraints. He proves that these solutions are quasi-periodic, i.e. they return to their initial shape up to a translation. Some numerical results due to Hyman correspond to this theoretical result (cf. [12] or [9]).

Our point of view is completely different. The matter is to find invariant measures on Hilbert spaces which are the phase spaces of Hamiltonian systems with at least two conservation laws. Next we use this construction to prove a Poisson's recurrence like theorem. Such a construction has first been carried out by Friedlander [6] for the wave equation with a cubic nonlinear- 
ity but some details in the proof seem obscure. It is also Zhidkov's point of view in different articles. In [25], he carries out this study for the equation $i u_{t}+u_{x x}+f\left(x,|u|^{2}\right) u=0$ where the nonlinearity is very weak. This study is generalized to the equation $i u_{t}+u_{x x}+|u|^{2} u=0$ in [27], except that the existence result of solutions to this equation in $L^{2}(0, A)$ can not be deduced from Tsutsumi's results in [19], who makes use of $L^{p}()-L^{q}()$ estimates which are not valid in the periodic case. On the other hand, we may now base the proof on a result of Bourgain [2]. In [28], Zhidkov studies in the same way the wave equation $u_{t t}-u_{x x}+f(x, u)=0$, once more for weak nonlinearities. The article [29] is the insertion of all former results in a wider frame of certain Hamiltonian systems.

The present paper is the enlightenment of this last article as well as the application to new classes of examples, in particular to numerical schemes. In connection with that we give a brief survey of hamiltonian schemes for approximating nonlinear partial differential equations of hamiltonian type. The outline is the following. In part 2, we construct invariant measures for Hamiltonian systems and we prove the Poisson's recurrence. The process we use is exactly the same as Zhidkov's but it's more explicit. Part 3 is devoted to the study of a few fields of application of this general theory. Finally part 4 deals particulary with recurrence in the case of schemes which is the phenomenon one actually observes during numerical simulations.

We may regret the fact that this kind of study does not fulfill the original aim. Indeed we do not really prove recurrence but the fact that the solutions come infinitely often near the initial data (with time intervals which may be not constant). Moreover the notion of proximity to the initial data is not the one we may commonly observe on numerical computations ; we test whether two neighborhoods (one for the initial data and one for the solution at time t) which may have a very complex structure have a nonempty intersection. In return, the results which are proved here may be applied to a far wider class of initial data than perturbations of spatially uniform solutions.

While finishing the drafting of this article we have been informed of some very similar work done by Bourgain (cf. [4]) for the nonlinear Schrödinger equation inspired by the work done by Lebowitz, Rose and Speer [14]. 


\section{Construction of invariant measures.}

The construction of invariant measures is made up of many steps. The first one consists in associating to the initial equation and the initial functional spaces projected equations on nested finite dimensional functional spaces. In these spaces we construct invariant Gaussian measures for the projected problem. The last step consists in passing to the limit as the dimension of the spaces tend to $+\infty$. This makes up the construction of so-called cylindrical measures on the whole of the phase space $X$.

\subsection{Setting the problem.}

We study the following Cauchy problem :

$$
\left\{\begin{array}{l}
\dot{u}(t)=J H^{\prime}(u(t)), \\
u\left(t_{0}\right)=\phi \in X
\end{array}\right.
$$

where $X$ is a Hilbert space such that $\mathcal{D}\left(=\mathcal{C}_{0}^{\infty}\right)$ is dense in $X^{*}$.

Let $Y$ be a Hilbert space which is dense in $X$, and let us assume that

the functional $H$ is $\mathcal{C}^{1}$ from $Y$ into,

the functional $J$ is linear from $X^{*}$ into $X$,

for all $g, h \in \mathcal{D}, g(J h)=-h(J g)$.

These properties imply that $H(u(t))$ does not depend on $t$. This yields a conservation law in $X$ for the system (1). Besides, we assume that $X$ may be endowed with a norm which is as well invariant. This is crucial in order to prove theorem 9 .

We split $H$ in two parts

$$
g(u)=H(u)-\frac{1}{2}(S u, u)_{X} .
$$

The part $g(u)$ has to contain all the nonlinearity of the initial equation and has to be defined for functions belonging to $X$.

The operator $S$ is assumed to be positive and self-adjoint on $X$ and $g$ defined on $X$, real valued and continuous.

We assume (H2.1) that we know how to solve the problem (1) in $X$ and that the solution is continuous with respect to the initial data, that is : for every $t_{0} \in, \varepsilon>0, T>0$, there exists $\delta>0$ such that

$$
\left\|u_{1}\left(t_{0}\right)-u_{2}\left(t_{0}\right)\right\|_{X}<\delta \Rightarrow\left\|u_{1}(t)-u_{2}(t)\right\|_{X}<\varepsilon
$$


for all $t \in I=\left[t_{0}-T, t_{0}+T\right]$.

We associate to this problem a sequence of finite dimensional problems. With that aim we construct a sequence of Hilbert subspaces of $Y$ :

$$
X_{1} \subset X_{2} \subset \ldots \subset X_{n} \subset \ldots \subset Y \subset X
$$

where $d_{n}<\infty$ is the dimension of the space $X_{n}$ and we assume that $\bigcup_{n} X_{n}$ is dense in $Y$.

We denote by $P_{n}$ the orthogonal projector from $X$ onto $X_{n}$, and we obtain a new problem set on this space:

$$
\left\{\begin{array}{l}
\dot{u}^{n}(t)=P_{n}\left[J H^{\prime}\left(P_{n} u^{n}(t)\right)\right] \\
u^{n}\left(t_{0}\right)=P_{n} \phi \in X_{n}
\end{array}\right.
$$

As in the case of (1), this system admits an invariant which is $H\left(P_{n} u^{n}(t)\right)$. We assume (H2.2) that $u^{n}(t)$ exists globally in time for every initial data $\phi \in X$ and that for all $t_{0} \in, \varepsilon>0, T>0$, there exists $\delta>0$ such that for all $n$

$$
\left\|u_{1}^{n}\left(t_{0}\right)-u_{2}^{n}\left(t_{0}\right)\right\|_{X}<\delta \Rightarrow\left\|u_{1}^{n}(t)-u_{2}^{n}(t)\right\|_{X}<\varepsilon
$$

for all $t \in I$. The fact that $\delta$ does not depend on $n$ is fundamental in the proof of theorem 8 .

We impose compatibility conditions over the different problems :

(H2.3) The solution $u^{n}$ to (2) converges to the solution $u$ to $(1)$ in $\mathcal{C}(I ; X)$ (uniformly with respect to $n$ (cf. theorem 8)).

Remark 1 The two former uniformity properties H2.2 and H2.3 imply the aforementionned property H2.1 of continuity with respect to the initial data for the problem (1).

(H2.4) The operator $S^{-1}$ is nuclear (which means for example that the sum of its eigenvalues is convergent) and maps $X_{n}$ into $X_{n}$.

(H2.5) The operator $J$ is defined on $X_{n}^{*}$ and $P_{n} J=J P_{n}^{*}$.

\subsection{Invariant measures in finite dimension.}

To begin with, we will construct an invariant measure for each finite dimensional system. The construction is based on the classical Liouville theorem 
(see for example Arnold [1]) :

Theorem 2 (Liouville) Let us consider the equation $\dot{z}=f(z)$ and $\rho(C)=\int_{C} \lambda(z) d z$ where $\lambda$ is a positive function and $C$ is a Borel set of ${ }^{m}$.

Then $\rho$ is invariant if and only if $\sum_{i=1}^{m} \frac{\partial}{\partial z_{i}}\left(\lambda f_{i}\right)=0$.

We will now carry out the construction of the invariant measure $\mu_{n}$ on the phase space of the finite dimensional system on $X_{n}$.

Let $\left(e_{1}, \ldots, e_{d_{n}}\right)$ be the eigenvectors of $S$ which generate $X_{n}$ (cf. $S^{-1}: X_{n} \rightarrow$ $\left.X_{n}\right)$ and let $F$ be a Borel set of ${ }^{d_{n}}$. Then we define the cylindrical set $M$ by :

$$
M=\left\{x \in X /\left[\left(x, e_{1}\right)_{X}, \ldots,\left(x, e_{d_{n}}\right)_{X}\right] \in F\right\} .
$$

Let $\mathcal{A}_{n}$ be the algebra whose elements are these cylinders and let $w_{n}$ be the function defined on $\mathcal{A}_{n}$ by

$$
w_{n}(M)=(2 \pi)^{-d_{n} / 2} \prod_{j=1}^{d_{n}} \lambda_{j}^{1 / 2} \int_{F} e^{-\frac{1}{2} \sum_{j=1}^{d_{n}} \lambda_{j} y_{j}^{2}} d y
$$

where $\lambda_{j}$ is the eigenvalue of $S$ corresponding to the eigenvector $e_{j}$.

In that way we do have constructed a measure $w_{n}$ on $\mathcal{A}_{n}$.

We set $u^{n}(t)=\sum_{j=1}^{d_{n}} a_{j}(t) e_{j}, a=\left(a_{1}, \ldots, a_{d_{n}}\right)$ and $h(a)=H\left(\sum_{j=1}^{d_{n}} a_{j}(t) e_{j}\right)$.

For every Borel set $A$ of ${ }^{d_{n}}$, we define the measure

$$
\mu_{n}^{\prime}(A)=(2 \pi)^{-d_{n} / 2} \prod_{j=1}^{d_{n}} \lambda_{j}^{1 / 2} \int_{A} e^{-h(a)} d a
$$

and notice that

$$
\dot{a}(t)=J \nabla_{a} h(a) .
$$

We apply Liouville's theorem with $\lambda(a)=e^{-h(a)}$, which yields the invariance of $\mu_{n}^{\prime}$ on the Borel sets of ${ }^{d_{n}}$. The inverse change of coordinates implies that $\mu_{n}$ is invariant on $\mathcal{A}_{n}$ where

$$
\mu_{n}(M)=(2 \pi)^{-d_{n} / 2} \prod_{j=1}^{d_{n}} \lambda_{j}^{1 / 2} \int_{M} e^{-H(u)} d u
$$


This ends the construction of invariant measures in finite dimensional spaces.

\subsection{Invariant measures in infinite dimension.}

Let $\mathcal{A}=\bigcup_{n} \mathcal{A}_{n}$. We associate to this new algebra $\mathcal{A}$ the minimal Borel $\sigma$ algebra $\mathcal{M}$ containing $\mathcal{A}$.

For each $M$ belonging to $\mathcal{M}$, we set $w_{n}(M)=w_{n}\left(M \cap X_{n}\right)$. This allows us to extend the measures $w_{n}$ over the whole of $\mathcal{M}$. Such a construction is licit since $M \cap X_{n}$ belongs to $\mathcal{M}$. The $\sigma$-additivity of the measure is conserved thanks to the following lemma (cf. Dalecky and Fomin [5]) and the fact that $w_{n}$ is a Gaussian measure with $S^{-1}$ as correlation operator.

Lemma 3 The measure $w_{n}$ is $\sigma$-additive on the algebra $\mathcal{M}$ if and only if $S^{-1}$ is a nuclear operator.

The proof of this lemma may be found in the appendix B.

We notice that for a fixed element $M$ in $\mathcal{A}$, and from a certain range $n$, the sequence $w_{n}(M)$ is constant. We take this value as the value of $w(M)$ and we extend $w$ over the $\sigma$-algebra $\mathcal{M}$.

Lemma 4 (Zhidkov) The sequence $\left\{w_{n}\right\}$ is weakly convergent to $w$ in $X$.

Proof. The only possible limit for $w_{n}$ is $w$ since $w_{n}(M)$ tends to $w(M)$ for each element $M$ of $\mathcal{A}$ and the extension to $\mathcal{M}$ is unique. There is also only left to prove that the sequence $\left\{w_{n}\right\}$ is weakly compact. For that aim we use Prohorov's theorem.

Theorem 5 (Prohorov) A subset $\mathcal{N}$ of the set of finite positive Borel measures on a complete separable metric space $(X, \rho)$ is precompact if and only if (i) there exists $M<\infty$, such that $\nu(X) \leq M$ for all $\nu \in \mathcal{N}$,

(ii) for all $\varepsilon>0$, there exists a compact set $K_{\varepsilon}$ in $X$, such that $\nu\left(X \backslash K_{\varepsilon}\right)<\varepsilon$ for all $\nu \in \mathcal{N}$.

We take $\mathcal{N}=\left\{w_{n}\right\}$. It is obvious that for all $n, w_{n}(X)=1$, therefore (i) is true.

Concerning (ii), the construction of the compact set $K_{\varepsilon}$ is carried out as follows.

Since $S^{-1}$ is a nuclear operator, $\operatorname{Tr} S^{-1}=\sum \lambda_{k}^{-1}<\infty$.

There exists a function $p$ defined on $\left[0, \infty\left[\right.\right.$ such that $\lim _{x \rightarrow+\infty} p(x)=+\infty$ and $\sum_{k} \lambda_{k}^{-1} p\left(\lambda_{k}\right)<\infty$ 
We set $T=p(S)$ and $Q=S^{-1} T$.

Hence $\operatorname{Tr} Q=\sum_{k} \lambda_{k}^{-1} p\left(\lambda_{k}\right)(<+\infty$ according to the assumption).

Let $B_{R}=\left\{u \in X /\left\|T^{1 / 2} u\right\|_{X} \leq R\right\}$ and $\mathcal{B}={\overline{\mathcal{B}_{\mathcal{R}}}}^{\mathcal{X}}$.

Let $\psi_{m}=\sum_{k} a_{k}^{m} \varphi_{k}$, be a sequence of elements of $B_{R}, \varphi_{k}$ denoting the eigenvectors of $S$.

Now $T^{1 / 2} \psi_{m}=\sum_{k} p\left(\lambda_{k}\right)^{1 / 2} a_{k}^{m} \varphi_{k}$ and $\left|T^{1 / 2} \psi_{m}\right|_{X}=\left|\sum_{k} p\left(\lambda_{k}\right)\left(a_{k}^{m}\right)^{2}\right|^{1 / 2} \leq R$.

In order to prove the compactness of $\mathcal{B}$, we only need to show that it is possible to make an estimate of the remainders of the series $\psi_{m}$ which is uniform in $m$.

Indeed $\sum_{k=K}^{+\infty}\left|a_{k}^{m}\right|^{2}=\sum_{k=K}^{+\infty} \frac{p\left(\lambda_{k}\right)\left(a_{k}^{m}\right)^{2}}{p\left(\lambda_{k}\right)}$.

We choose $K$ such that for all $k \geq K, \frac{1}{p\left(\lambda_{k}\right)} \leq \frac{R}{\varepsilon}$.

This shows that for all $\varepsilon$, there exists an integer $K$ such that for all $m$

$$
\left|\sum_{k=K}^{+\infty} a_{k}^{m} \varphi_{k}\right|_{X}^{2} \leq \varepsilon
$$

Then $\mathcal{B}$ is compact for all $R$.

Now, (cf. Lemma B.2 in Appendix B) for a Gaussian measure $\mu_{B}$ with $B$ as correlation operator we have $\mu_{B}\left\{x:(A x, x)_{X} \geq 1\right\} \leq \operatorname{Tr} A B$.

We take $B=P_{n} S^{-1}$ and $A=T$, hence

$$
\begin{aligned}
w_{n}\left\{x /(T u, u)_{X} \geq 1\right\} & \leq \operatorname{Tr} P_{n} S^{-1} T \leq \operatorname{Tr} Q, \\
w_{n}(X \backslash B) \leq w_{n}\left(X \backslash B_{R}\right)=w_{n}\left\{x /(T u, u)_{X} \geq R\right\} & \leq \frac{\operatorname{Tr} Q}{R^{2}} .
\end{aligned}
$$

Therefore $w_{n}(X \backslash B) \leq \frac{\operatorname{Tr} Q}{R^{2}}$.

For each $\varepsilon$, we set $R=\sqrt{\frac{\operatorname{Tr} Q}{\varepsilon}}$ and $K_{\varepsilon}=B$.

Consequently and thanks to Prohorov's theorem, $w_{n}$ is weakly compact and $w_{n}$ tends weakly to the only possible limit, $w$.

As in the case of $w_{n}$, we set $\mu_{n}(M)=\mu_{n}\left(M \cap X_{n}\right)$, for each element $M$ of $\mathcal{M}$. For each Borel set $\Omega$ of $X$, let us set

$$
\mu(\Omega)=\int_{\Omega} e^{-g(u)} w(d u)
$$


Then we get the following result

Lemma 6 Let $\Omega$ be an opened set of $X$ such that $\mu(\Omega)<\infty$. Then

$$
\liminf _{n \rightarrow \infty} \mu_{n}(\Omega) \geq \mu(\Omega)
$$

Proof. Let $\Omega$ be an open Borel set of $X$, then for every $\varepsilon$, there exists a function $\theta$ defined on $X$, with $0 \leq \theta(u) \leq 1$, such that

$$
\int_{\Omega} \theta(u) e^{-g(u)} w(d u) \geq \mu(\Omega)-\varepsilon .
$$

Let us set $M=\Omega \cap X_{n}$ and let $F$ be the Borel set of ${ }^{d_{n}}$ which is associated to $M$.

$$
\begin{aligned}
\mu_{n}(\Omega) & =(2 \pi)^{-d_{n} / 2} \prod_{j=1}^{d_{n}} \lambda_{j}^{1 / 2} \int_{F} e^{-h(y)} d y, \\
& =\int_{F} e^{-g\left(\sum_{j=1}^{d_{n}} y_{j} e_{j}\right)}(2 \pi)^{-d_{n} / 2} \prod_{j=1}^{d_{n}} \lambda_{j}^{1 / 2} e^{-\frac{1}{2} \lambda_{j} y_{j}^{2}} d y, \\
& =\int_{\Omega} e^{-g(u)} w_{n}(d u) .
\end{aligned}
$$

$$
\begin{aligned}
\liminf _{n \rightarrow \infty} \mu_{n}(\Omega) & =\liminf _{n \rightarrow \infty} \int_{\Omega} e^{-g(u)} w_{n}(d u), \\
& \geq \liminf _{n \rightarrow \infty} \int_{\Omega} \theta(u) e^{-g(u)} w_{n}(d u), \\
& =\int_{\Omega} \theta(u) e^{-g(u)} w(d u), \\
& \geq \mu(\Omega)-\varepsilon .
\end{aligned}
$$

Letting $\varepsilon$ tend to 0 , we obtain $\liminf _{n \rightarrow \infty} \mu_{n}(\Omega) \geq \mu(\Omega)$.

Corollary 7 Let $\Phi$ be a closed set of $X$. Then

$$
\limsup _{n \rightarrow \infty} \mu_{n}(\Phi) \leq \mu(\Phi)
$$

We finally state the theorem for the invariance of measure $\mu$. We denote 
$f(\phi, t)=u\left(t+t_{0}\right)$ where $u$ is the solution to $(1)$,

$f_{n}(\phi, t)=u^{n}\left(t+t_{0}\right)$ where $u^{n}$ is the solution to $(2)$.

Theorem 8 Let $\Omega$ be an opened set of $X$ and $\Omega_{t}=f(\Omega, t)$. Under asumptions (H2.1) to (H2.5), we have the property

$$
\mu(\Omega)=\mu\left(\Omega_{t}\right)
$$

Proof. The continuity assumption with respect to the initial data yields that $\Omega_{t}$ is as well an open set of $X$. We only make the proof in the case when $\mu(\Omega)$ and $\mu\left(\Omega_{t}\right)$ are assumed to be finite. Then for all $\varepsilon>0$, there exists a compact set $K$ such that $\mu(\Omega \backslash K) \leq \varepsilon$. It is obvious that $K_{t} \subset \Omega_{t}$ is compact.

Let $\alpha=\min \left\{\operatorname{dist}(K, \partial \Omega) ; \operatorname{dist}\left(K_{t}, \partial \Omega_{t}\right)\right\}$. For each element $u$ in $K$, there exists an open ball $B(u)$ with center $u \in \Omega$ such that $\operatorname{dist}\left(f_{n}(u, t) ; f_{n}(v, t)\right)<\frac{\alpha}{3}$, for all $v \in B(u)$ and for all $n$ according to the continuity assumption with respect to the initial data for the problem (2).

We set $\Omega_{\beta}=\left\{v \in \Omega_{t} / \operatorname{dist}\left(v, \partial \Omega_{t}\right) \geq \beta\right\}$, and we choose a finite covering $B\left(u_{1}\right)$, $\ldots, B\left(u_{l}\right)$ of $K$. We set $D=\bigcup_{i=1}^{l} B\left(u_{i}\right)$. Since $u^{n}(t)$ converges uniformly with respect to $n$ to $u(t), f_{n}(D, t) \subset \Omega_{\frac{\alpha}{4}}$ for a sufficiently large $n$.

$$
\begin{aligned}
\mu(\Omega) & \leq \mu(D)+\varepsilon \\
& \leq \liminf _{n \rightarrow \infty} \mu_{n}(D)+\varepsilon, \\
& \leq \liminf _{n \rightarrow \infty} \mu_{n}\left(D \cap X_{n}\right)+\varepsilon, \\
& \leq \liminf _{n \rightarrow \infty} \mu_{n}\left(f_{n}\left(D \cap X_{n}, t\right)\right)+\varepsilon, \\
& \leq \limsup _{n \rightarrow \infty} \mu_{n}\left(\Omega_{\frac{\alpha}{4}}\right)+\varepsilon, \\
& \leq \mu\left(\Omega_{t}\right)+\varepsilon .
\end{aligned}
$$

Hence $\mu(\Omega) \leq \mu\left(\Omega_{t}\right)$, and since time has no privileged direction, $\mu(\Omega)=$ $\mu\left(\Omega_{t}\right)$.

\subsection{Poisson's Recurrence.}

Theorem 9 For almost every initial data $\phi$, the trajectory $f(\phi, t)$ is Poisson recurrent. 
Proof. Thanks to theorem 8, for each neighborhood $\mathcal{V}$ of the initial data, $\mu(\mathcal{V})=\mu\left(\mathcal{V}_{t}\right)$.

Let $B\left(x_{i}, \varepsilon\right)$ be a finite covering of the phase space which is weakly compact thanks to the first conserved quantity. There exists an increasing sequence $t_{n}$ tending to infinity and $x_{i}$ such that $\mu\left(\mathcal{V}_{t_{n}} \cap B\left(x_{i}, \varepsilon\right)\right)>0$.

Let $K$ be a compact subset of the union of the $\mathcal{V}_{t_{n}}$. There exists a finite sequence $t_{n_{i}}$ such that $\left\{\mathcal{V}_{t_{n_{i}}}\right\}$ is a finite covering of $K$. Hence there exists an index $j$ and a subsequence $t_{n}^{\prime}$ of $t_{n}$ such that $\mu\left(\mathcal{V}_{t_{n_{j}}} \cap \mathcal{V}_{t_{n}^{\prime}}\right)>0$. Let us set $\tilde{t}_{n}=t_{n}^{\prime}-t_{n_{j}}$, this defines a sequence which tends to infinity and such that $\mu\left(\mathcal{V} \cap \mathcal{V}_{\tilde{t}_{n}}\right)>0$. So the solution comes for almost every initial data infinitely often near its initial value.

Remark 10 This last argument is still valid in the case of schemes with a time discretisation (cf. Part 4).

This last theorem is the only one which uses an invariant quantity in $X$. The construction of measures holds even if this condition is not fulfilled. Theorem 9 is also valid in the case where the total measure is finite $(\mu(X)<\infty)$. 


\section{Applications.}

Different classical equations may be written in the form (1). This is for example the case of the Schrödinger or the wave equations.

These applications are nevertheless limited by the following facts :

* The nonlinearity of the equation has to be defined for functions belonging to $X$.

* The space $X$ endowed by the norm which is the invariant defined for the less regular functions has to be a Banach space.

* The operator $S^{-1}$ has to be nuclear.

Each of the following examples will be explained in two steps :

- the setting up of the equation into an Hamiltonian form. We determine explicitly the invariants of the equation, the spaces $X$ and $Y$, the operators $J$ and $S$ as well as the functional $g$.

In this section we will have to check the following hypothesis :

(H3.1) $X$ and $Y$ are Hilbert spaces, and we have appropriate density results,

(H3.2) $H: Y \rightarrow$ is $\mathcal{C}^{1}$,

(H3.3) $J: X^{*} \rightarrow X$ is linear and skew-adjoint,

(H3.4) there is at least one invariant norm,

(H3.5) $g(u)$ is continuous and defined on $X$,

(H3.6) $S>0$ is self-adjoint,

(H3.7) $S^{-1}$ is nuclear,

(H3.8) $J: X_{n}^{*} \rightarrow X_{n}$ and $P_{n} J=J P_{n}$;

- the testing of the remaining hypotheses, that is :

(H3.9) there exist solutions in $X$ and $X_{n}$,

(H3.10) these solutions are continuous with respect to the initial data.

(H3.11) $u_{n}$ tends to $n$ in $\mathcal{C}(I ; X)$.

Since the study of Cauchy problems is not the aim of this article, we only refer to other articles for the testing of these hypotheses.

\subsection{Setting up.}

\subsubsection{The nonlinear Schrödinger equation.}

We consider the problem

$$
\left\{\begin{array}{l}
i u_{t}+\Delta u+f\left(x,|u|^{2}\right) u=0, \quad x \in(0, A), t \in \\
u(0, t)=u(A, t) \\
u\left(x, t_{0}\right)=u_{0}(x)
\end{array}\right.
$$

We transform this problem setting $u=\left(u_{1}, u_{2}\right)$. 
The partial differential equation becomes

$$
\left\{\begin{array}{l}
u_{1 t}+\Delta u_{2}+f\left(x,\left(u_{1}\right)^{2}+\left(u_{2}\right)^{2}\right) u_{2}=0 \\
u_{2 t}-\Delta u_{1}-f\left(x,\left(u_{1}\right)^{2}+\left(u_{2}\right)^{2}\right) u_{1}=0
\end{array}\right.
$$

We set $F(x, s)=\frac{1}{2} \int_{0}^{s} f(x, \sigma) d \sigma$. We know two invariants for this equation

$$
\begin{aligned}
& E\left(u_{1}, u_{2}\right)=\frac{1}{2} \int_{0}^{A}\left\{\left(u_{1}\right)^{2}+\left(u_{2}\right)^{2}\right\} d x, \\
& H\left(u_{1}, u_{2}\right)=\int_{0}^{A}\left\{\frac{1}{2}\left(\left(\nabla u_{1}\right)^{2}+\left(\nabla u_{2}\right)^{2}\right)-F\left(x,\left(u_{1}\right)^{2}+\left(u_{2}\right)^{2}\right)\right\} d x .
\end{aligned}
$$

The gradient of this last invariant is :

$$
H^{\prime}\left(u_{1}, u_{2}\right)=\left(\begin{array}{l}
-\Delta u_{1}+f\left(x,\left(u_{1}\right)^{2}+\left(u_{2}\right)^{2}\right) u_{1} \\
-\Delta u_{2}+f\left(x,\left(u_{1}\right)^{2}+\left(u_{2}\right)^{2}\right) u_{2}
\end{array}\right)
$$

The functional spaces we will consider are $X=L^{2} \times L^{2}$ and $Y=H^{1} \times H^{1}$, the operators $J$ et $S$ being respectively equal to

$$
J=\left(\begin{array}{cc}
0 & I \\
-I & 0
\end{array}\right) \quad \text { and } \quad S=\left(\begin{array}{cc}
-\Delta & 0 \\
0 & -\Delta
\end{array}\right)
$$

This allows us to compute

$$
g\left(u_{1}, u_{2}\right)=-\int_{0}^{A} F\left(x,\left(u_{1}\right)^{2}+\left(u_{2}\right)^{2}\right) d x .
$$

\subsubsection{The wave equation.}

We consider the problem

$$
\left\{\begin{array}{l}
u_{t t}-u_{x x}+f(x, u)=0, \quad x \in(0, A), t \in \\
u(0, t)=u(A, t), \\
u\left(x, t_{0}\right)=u_{0}(x), \quad u_{t}\left(x, t_{0}\right)=v_{0}(x)
\end{array}\right.
$$


We transform this problem setting $v=u_{t}$.

The partial differential equation becomes

$$
\left\{\begin{aligned}
u_{t}-v & =0 \\
v_{t}-u_{x x}+f(x, u) & =0
\end{aligned}\right.
$$

We set $F(x, u)=\frac{1}{2} \int_{0}^{u} f(x, s) d s$, and we may find an invariant in the form :

$$
H(u, v)=\int_{0}^{A}\left\{\frac{1}{2}\left((v)^{2}+\left(u_{x}\right)^{2}\right)+F(x, u)\right\} d x
$$

The computation of its gradient yields :

$$
H^{\prime}(u, v)=\left(\begin{array}{c}
-\Delta u+f(x, u) \\
v
\end{array}\right)
$$

We consider the functional spaces $X=L^{2} \times H^{-1}$ and $Y=H^{1} \times L^{2}$. The operators $J$ and $S$ are respectively equal to

$$
J=\left(\begin{array}{cc}
0 & I \\
-I & 0
\end{array}\right) \quad \text { and } \quad S=\left(\begin{array}{cc}
-\Delta & 0 \\
0 & -\Delta
\end{array}\right)
$$

The computation of $g$ gives

$$
g(u, v)=\int_{0}^{A} F(x, u) d x
$$

\subsection{Testing the hypotheses.}

\subsubsection{The nonlinear Schrödinger equation.}

The global existence of solutions to (1) for an initial data in $L^{2}$ is given in an article by Bourgain [2] and the conditions that should be imposed on the nonlinearity $f$ are studied in the appendix A. Concerning the finite dimensional 
problem (2), it may be written in the form

$$
\dot{u}^{n}=P_{n}\left(\begin{array}{cc}
0 & I \\
-I & 0
\end{array}\right)\left(\begin{array}{l}
-\Delta u_{1}^{n}+f\left(x,\left(u_{1}^{n}\right)^{2}+\left(u_{2}^{n}\right)^{2}\right) u_{1}^{n} \\
-\Delta u_{2}^{n}+f\left(x,\left(u_{1}^{n}\right)^{2}+\left(u_{2}^{n}\right)^{2}\right) u_{2}^{n}
\end{array}\right) .
$$

Since $X_{n}$ is supposed to be stable with respect to $S$, the operator representing the linear part is the same one and the estimates obtained for the continuous case are still valid. The assumption on the nonlinear part still holds. Hence there exists a unique global solution.

The continuity with respect to the initial data may be studied in a classical way thanks to estimates which are analogous to those of the proof for the local existence.

The nonlinearities we choose here verify for example

$$
\begin{aligned}
& \left\|f\left(x,|u|^{2}\right)\right\|_{2} \leq C \sum_{\gamma}\|u\|_{4}^{\gamma} \\
& \text { and }\left\|\nabla_{s} f\left(x,|u|^{2}\right)\right\|_{4} \leq C \sum_{\eta} \sup _{u \in B(0, M)}\|u\|_{4}^{\eta} \text { when } u \in B(0, M),
\end{aligned}
$$

the sum over $\gamma$ and $\eta$ dealing with a finite number of terms with $0 \leq \gamma$ and $-1 \leq \eta$.

These conditions are weaker than those initialy chosen by Zhidkov (cf. [29]), that is

$$
|f(x, s)|+\left|(1+s) \nabla_{s} f(x, s)\right|<C \text { for all } x, s .
$$

He also carries out the application of the method to the cubic nonlinear Schrödinger equation. In the present paper our aim is to find the weakest assumptions under which the construction is possible. The method used by Zhidkov for the cubic Schrödinger equation is slighlty different. He defines a sequence of Schrödinger equations with very weak nonlinearities which tend in a certain sense to the cubic nonlinearity. He constructs an invariant measure for each of these equations and then passes to the limit over the measures. This yields an invariant measure for the cubic Schrödinger equation.

\subsubsection{The wave equation.}

Here we will keep Zhidkov's hypotheses on the nonlinearity, that is

$$
|f(x, u)| \leq C\left(1+u^{2}\right)^{1 / 2}
$$


and

$$
\left|\partial_{u} f(x, u)\right| \leq C .
$$

We may remark that these conditions are non fulfiled in the case of the cubic wave equation which is the case studied by Friedlander [6].

We refer to Zhidkov's article [28] for the proof of the wellposedness for this this equation. The wellposedness for the discretized equation is made with the same type of arguments than in the case of the nonlinear Schrödinger equation.

\subsection{Why this approach fails in the Korteweg-de Vries equation case.}

In [29] Zhidkov treats the case of a Korteweg-de Vries equation, but it is a linear one :

$$
u_{t}+(a(x) u)_{x}+u_{x x x}=0 .
$$

This induces us to study the usual Korteweg-de Vries equation. We will show why it's is impossible to apply the general theory to this problem. We will also consider the equation

$$
\left\{\begin{array}{l}
u_{t}+u_{x x x}+u^{k} u_{x}=0, \quad x \in(0, A), t \in \\
u(0, t)=u(A, t) \\
u\left(x, t_{0}\right)=u_{0}(x) .
\end{array}\right.
$$

This equation has the following invariants :

$$
\begin{aligned}
& H_{0}(u)=\int_{0}^{A} u d x \\
& H_{1}(u)=\frac{1}{2} \int_{0}^{A} u^{2} d x \\
& H_{2}(u)=\int_{0}^{A}\left\{\frac{1}{2} u_{x}^{2}-\frac{1}{(k+1)(k+2)} u^{k+2}\right\} d x,
\end{aligned}
$$

and in the case when $k=1$, there are some additional invariants, the first one 
being

$$
H_{3}(u)=\int_{0}^{A}\left\{\frac{1}{2} u_{x x}^{2}-\frac{5}{6} u u_{x}^{2}+\frac{5}{72} u^{4}\right\} d x .
$$

Their gradients are respectively equal to

$$
\begin{aligned}
& H_{1}^{\prime}(u)=u \\
& H_{2}^{\prime}(u)=-u_{x x}-\frac{1}{(k+1)} u^{k+1}, \\
& H_{3}^{\prime}(u)=u_{x x x x}+\frac{5}{6} u_{x}^{2}+\frac{5}{2} u u_{x x}+\frac{5}{18} u^{3} .
\end{aligned}
$$

The following problem faces us. The nonlinear part imposes $X$ to be included in $H^{1}$. Since we dispose of various invariants we could take $H_{3}$ as Hamiltonian (in the case $k=1$ ), unfortunately it is not possible to set the Korteweg-de Vries equation in a Hamiltonian form with this invariant. We know of two hamiltonian forms for the Korteweg-de Vries equation. The more classical one is the following :

$$
u_{t}=\partial_{x}\left(u_{x x}+\frac{1}{2} u^{2}\right)
$$

which is associated to $H_{2}$ taking $J_{2}=-\partial_{x}, S_{2}=-\Delta$ and $g_{2}(u)=-\int_{0}^{A} \frac{1}{(k+1)(k+2)}|u|^{k+2} d x$.

An other hamiltonian form is described by Olver [17] :

$$
u_{t}=\left(\partial_{x}^{3}+\frac{2}{3} u \partial_{x}+\frac{1}{3} u_{x}\right) u
$$

this time considering $H_{1}$ as hamiltonian and taking $J_{1}=-\left(\partial_{x}^{3}+\frac{2}{3} u \partial_{x}+\frac{1}{3} u_{x}\right)$, $S_{1}=-I$. and $g_{1}(u)=0$.

None of those two forms is compatible with our different assumptions on the different operators. 


\section{Particular case of numerical schemes.}

In the case of schemes, only the finite dimensional construction is useful. The choice of spaces becomes now indifferent since all norms are equivalent. This solves also the limitations on possible nonlinearities and the operator $S^{-1}$ becomes necessarily nuclear. In the case when $S$ is singular, we have to consider its reduction to the orthogonal space of its kernel and choose a nonlinearity such that the dynamical systems preserves this subspace. In the examples presented here $S$ is a discretization of the Laplacian which happens to be singular in the periodic case (the kernel is the constants) and nonsingular in the zero boundary case.

We will now consider equations with two invariants and try to find a discretization which conserves analogous invariants. That way we may hope that the observed phenomena which are connected to the existence of invariants for the discretized equation may extend to the continuous case. The case of semi-discretizations in space by a Galerkin method in bases of eigenvectors of $S$ has already been treated in the theoretical part (cf. Part 2.2). We will produce here other types of discretizations.

\subsection{The nonlinear Schrödinger equation.}

We remind that the cubic Schrödinger equation

$$
u_{t}=i u_{x x}+i q|u|^{2} u
$$

considered on the interval $\left[-\frac{L}{2}, \frac{L}{2}\right]$ with periodic boundary conditions admits two classical invariants :

$$
\begin{aligned}
& \frac{1}{2} \int_{-\frac{L}{2}}^{\frac{L}{2}}|u|^{2} d x=c_{1}, \\
& \frac{1}{2} \int_{-\frac{L}{2}}^{\frac{L}{2}}\left(\left|u_{x}\right|^{2}-\frac{1}{2} q|u|^{4}\right) d x=c_{2} .
\end{aligned}
$$

\subsubsection{Space discretizations.}

In what follows we will denote by $\delta$ the forward-space derivative

$$
\delta U_{j}=h^{-1}\left(U_{j+1}-U_{j}\right)
$$


and $\delta^{2}$ the second central-space derivative

$$
\delta^{2} U_{j}=h^{-2}\left(U_{j+1}-2 U_{j}+U_{j-1}\right) .
$$

The indexes for the space variable will be $j$, for the Fourier variable $k$ and the superscript for the time variable will be $n$.

We discretise $\left[-\frac{L}{2}, \frac{L}{2}\right]$ in $K$ equal intervals. Let $h=\frac{L}{K}$.

We consider here three types of space discretizations : finite difference schemes, spectral schemes and pseudo-spectral schemes.

Finite difference scheme.

Let $U_{j}(t)$ be an approximation of $u\left(-\frac{L}{2}+(j-1) h, t\right)$. The most classical finite difference scheme is given by

$$
\dot{U}_{j}=i \delta^{2} U_{j}+i q\left|U_{j}\right|^{2} U_{j}
$$

Spectral scheme.

We make the following Ansatz : $u$ is reduced to a finite number of frequences, i.e. it may be written in the form

$$
u(x, t)=\sum_{-K / 2}^{K / 2} A_{k}(t) \exp \left(i \mu_{k} x\right) .
$$

Hence we obtain

$$
\dot{A}_{k}=-i \mu_{k}^{2} A_{k}+i q \sum_{l_{1}+l_{2}-l_{3}=k} A_{l_{1}} A_{l_{2}} A_{l_{3}}^{*} .
$$

Pseudo-spectral scheme.

It is based on the fact that we may use a FFT to solve the problem numerically. Therefore we define an analogue of the Fourier transform

$$
F_{k} U_{j}=A_{k}=\frac{1}{K} \sum_{-K / 2}^{(K / 2)-1} U_{j} \exp \left(-i \mu_{k} x_{j}\right),
$$

and of the inverse Fourier transform

$$
F_{j}^{-1} A_{k}=U_{j}=\sum_{-K / 2}^{(K / 2)-1} A_{k} \exp \left(i \mu_{k} x_{j}\right) .
$$

The equation may also be discretised using

$$
\dot{A}_{k}=-i \mu_{k}^{2} A_{k}+i q F_{k}\left(U_{j}\left|U_{j}\right|^{2}\right)
$$


or equivalently

$$
\dot{U}_{j}=-i F_{k}^{-1}\left(\mu_{k}^{2} F_{k}\left(U_{j}\right)\right)+i q\left|U_{j}\right|^{2} U_{j}
$$

A variant of the finite difference scheme is the following (cf. Herbst and Ablowitz [8]).

Integrable scheme.

$$
\dot{U}_{j}=i \delta^{2} U_{j}+i \frac{1}{2} q\left|U_{j}\right|^{2}\left(U_{j+1}+U_{j-1}\right)
$$

\subsubsection{Analysis of space discretizations.}

Finding two conservation laws allows the use of the general theory for these schemes. Each of the three first schemes have two invariant quantities which are similar to those of equation (1). The setting in an hamiltonian form according to the notations of Part 2 is very similar to the continuous case.

Finite difference scheme.

$$
\begin{aligned}
& \frac{h^{(K / 2)-1}}{\sum_{-K / 2}}\left|U_{j}\right|^{2}=C_{1,1} \\
& \frac{h^{2}}{\sum_{-K / 2}^{(K / 2)-1}}\left\{\left|\delta U_{j}\right|^{2}-\frac{1}{2} q\left|U_{j}\right|^{4}\right\}=C_{1,2} .
\end{aligned}
$$

We set $U=\left(V_{-K / 2}, \ldots, V_{(K / 2)-1}, W_{-K / 2}, \ldots, W_{(K / 2)-1}\right)^{T}$ where $U_{j}=V_{j}+i W_{j}$. The scheme (6) becomes

$$
\left\{\begin{array}{l}
\dot{V}_{j}=-\delta^{2} W_{j}-q\left(V_{j}^{2}+W_{j}^{2}\right) W_{j} \\
\dot{W}_{j}=\delta^{2} V_{j}+q\left(V_{j}^{2}+W_{j}^{2}\right) V_{j} .
\end{array}\right.
$$

Then $J=\left(\begin{array}{cc}0 & h I_{K} \\ -h I_{K} & 0\end{array}\right)$ and $S=\left(\begin{array}{cc}-D_{K}^{2} & 0 \\ 0 & -D_{K}^{2}\end{array}\right)$ 
where $D_{K}^{2}=\frac{1}{h^{2}}\left(\begin{array}{cccc}-2 & 1 & & 1 \\ 1 & \ddots & \ddots & \\ & \ddots & \ddots & 1 \\ 1 & & 1 & -2\end{array}\right)$

and $H^{\prime}(U)=\left(\ldots, \delta^{2} V_{j}+q\left(V_{j}^{2}+W_{j}^{2}\right) V_{j}, \ldots, \delta^{2} W_{j}+q\left(V_{j}^{2}+W_{j}^{2}\right) W_{j}, \ldots\right)^{T}$.

Spectral scheme.

$$
\begin{aligned}
& \frac{L}{2} \sum_{-K / 2}^{K / 2}\left|A_{k}\right|^{2}=C_{2,1} \\
& \frac{L}{2}\left\{\sum_{-K / 2}^{K / 2} \mu_{k}^{2}\left|A_{k}\right|^{2}-\frac{1}{2} q \sum_{-K / 2 \leq l_{1}, l_{2}, l_{3} \leq K / 2} A_{l_{1}} A_{l_{2}} A_{l_{3}}^{*} A_{l_{1}+l_{2}-l_{3}}^{*}\right\}=C_{2,2}
\end{aligned}
$$

Pseudo-spectral scheme.

$$
\begin{aligned}
& \frac{h^{2}}{(K / 2)-1}\left|U_{j}\right|^{2}=C_{3,1}, \\
& \frac{L}{2} \sum_{k=-K / 2}^{(K / 2)-1} \mu_{k}^{2}\left|A_{k}\right|^{2}-\frac{1}{4} q h \sum_{j=-K / 2}^{(K / 2)-1}\left|U_{j}\right|^{4}=C_{3,2} .
\end{aligned}
$$

Integrable scheme.

In the case of the integrable scheme we may also find two invariants but the second one does not seem to have a counterpart at the level of the continuous equation :

$$
\begin{aligned}
& \frac{h^{(K / 2)-1}}{\sum_{-K / 2}}\left|U_{j}\right|^{2}=C_{4,1} \\
& \frac{h^{(K / 2)-1}}{2} \sum_{-K / 2}^{(K}\left\{U_{j}^{*}\left(U_{j+1}+U_{j-1}\right)-4 q^{-1} \ln \left(1+\frac{1}{2} q\left|U_{j}\right|^{2}\right)\right\}=C_{4,2} .
\end{aligned}
$$

Remark 1 An other proof of recurrence.

A direct and straightforward proof of the periodicity of these three schemes for a small number of modes as well as the expression of the recurrence time thanks to an elliptic integral may be found in the article of Weideman and Herbst [20]. 


\subsubsection{A full discretization.}

For the effective computation of the former schemes we have to choose a time discretization which has to conserve the different quantities. We may use the mid-point scheme which is a finite difference scheme in both the time and the space variables :

$$
\begin{aligned}
& \frac{u_{j}^{n+1}-u_{j}^{n}}{\Delta t}=i \frac{u_{j+1}^{n}-2 u_{j}^{n}+u_{j-1}^{n}}{2 h^{2}}+i \frac{u_{j+1}^{n+1}-2 u_{j}^{n+1}+u_{j-1}^{n+1}}{2 h^{2}} \\
& +i \frac{q}{4}\left(\left|u_{j}^{n}\right|^{2}+\left|u_{j}^{n+1}\right|^{2}\right)\left(u_{j}^{n}+u_{j}^{n+1}\right) .
\end{aligned}
$$

\subsubsection{Analysis of the full discretization.}

Two quantities are conserved

$$
\begin{aligned}
& \frac{h^{2}}{2} \sum_{-K / 2}^{(K / 2)-1}\left|u_{j}^{n}\right|^{2}=\frac{h}{2} \sum_{-K / 2}^{(K / 2)-1}\left|u_{j}^{n+1}\right|^{2} \\
& \frac{h^{2}}{2} \sum_{-K / 2}^{(K / 2)-1}\left(\left|\delta u_{j}^{n}\right|^{2}-\frac{1}{2} q\left|u_{j}^{n}\right|^{4}\right)=\frac{h}{2} \sum_{-K / 2}^{(K / 2)-1}\left(\left|\delta u_{j}^{n+1}\right|^{2}-\frac{1}{2} q\left|u_{j}^{n+1}\right|^{4}\right) .
\end{aligned}
$$

Recurrence has been actually observed for this scheme but it seems nevertheless impossible to apply the above theory to such full discretized schemes, indeed setting for example $U^{n}=\left(v_{-K / 2}^{n}, \ldots, v_{(K / 2)-1}^{n}, w_{-K / 2}^{n}, \ldots, w_{(K / 2)-1}^{n}\right)^{T}$ where $u_{j}^{n}=v_{j}^{n}+i w_{j}^{n}$ we may use the same operator $S$ as in the space discretization case. On the other hand it is not possible to find an operator $J$ satisfying

$$
\frac{U^{n+1}-U^{n}}{\Delta t}=J H^{\prime}\left(U^{n+1}\right)
$$

This is the only restriction since we noticed (cf. the end of part 2) that Theorem 9 also holds for schemes with a time discretization.

\subsection{The wave equation.}

\subsubsection{A space discretization.}

Let us consider a nonlinear wave equation in the form :

$$
u_{t t}-u_{x x}+f(u)=0
$$


A space finite difference discretization is possible :

$$
\ddot{u}_{j}-\delta^{2} u_{j}+f\left(u_{j}\right)=0 \text {. }
$$

Setting $F(s)=\frac{1}{2} \int_{0}^{s} f(\sigma) d \sigma$, we obtain the following invariant quantity for (13) :

$$
\sum_{-K / 2}^{(K / 2)-1}\left\{\frac{1}{2}\left(\left|\dot{u}_{j}\right|^{2}+\left|\delta u_{j}\right|^{2}\right)+F\left(u_{j}\right)\right\}=C .
$$

Let $v_{j}=\dot{u}_{j}$,

setting $U=\left(u_{-K / 2}, \ldots, u_{(K / 2)-1}\right)^{T}$ and $V=\left(v_{-K / 2}, \ldots, v_{(K / 2)-1}\right)^{T}$, we set the discretized wave equation in the the hamiltonian form using

$$
\begin{gathered}
H(U, V)=-\frac{1}{2}\left(D_{K}^{2} U, U\right)+\frac{1}{2}(V, V)+F(U) \\
H^{\prime}(U, V)=\left(\begin{array}{c}
-D_{K}^{2} U+f(U) \\
V
\end{array}\right), J=\left(\begin{array}{cc}
0 & h I_{K} \\
-h I_{K} & 0
\end{array}\right), S=\left(\begin{array}{cc}
-D_{K}^{2} & 0 \\
0 & I_{K}
\end{array}\right) .
\end{gathered}
$$

\subsubsection{A full discretization.}

To obtain an invariant for a full finite difference scheme for the wave equation, we have to restrict ourselves once more to a smaller family of nonlinearities that is $f(u)=A u^{2^{m}-1}$. Then we use for example the scheme :

$$
\frac{u_{j}^{n+1}-2 u_{j}^{n}+u_{j}^{n-1}}{\Delta t^{2}}-\frac{1}{2} \delta^{2} u_{j}^{n+1}-\frac{1}{2} \delta^{2} u_{j}^{n-1}+\frac{A}{2^{m}} \sum_{l=0}^{2^{m}-1}\left(u_{j}^{n+1}\right)^{2^{m}-1-l}\left(u_{j}^{n-1}\right)^{l}=0 .
$$

For this scheme the invariant quantity is :

$$
\sum_{-K / 2}^{(K / 2)-1}\left\{\frac{\left|u_{j}^{n-1}-u_{j}^{n}\right|^{2}}{\Delta t^{2}}+\frac{1}{2}\left(\left|\delta u_{j}^{n+1}\right|^{2}+\left|\delta u_{j}^{n}\right|^{2}\right)+\frac{A}{2^{m}}\left(\left(u_{j}^{n+1}\right)^{2^{m}}+\left(u_{j}^{n}\right)^{2^{m}}\right)\right\}=C .
$$

In this case we are facing the same problem for the setting in an hamiltonian form as in the case of the full discretization of the cubic nonlinear Schrödinger equation. 


\subsection{The Zakharov equations.}

\subsubsection{The continuous equations.}

In the context of plasma physics, Zakharov [24] has introduced the following system

$$
\left\{\begin{array}{l}
i E_{t}+E_{x x}=N E \\
N_{t t}-N_{x x}=\frac{\partial^{2}}{\partial x^{2}}\left(|E|^{2}\right) .
\end{array}\right.
$$

Setting $M=N_{t}$ and $\sqrt{2} E=F+i G$, we obtain the following formulation.

$$
\left\{\begin{array}{l}
F_{t}=-G_{x x}+N G \\
G_{t}=F_{x x}-N F \\
N_{t}=M \\
M_{t}=N_{x x}+\frac{1}{2} \frac{\partial^{2}}{\partial x^{2}}\left(F^{2}+G^{2}\right) .
\end{array}\right.
$$

Considering these equations with periodic boundary conditions on the interval $\left[-\frac{L}{2}, \frac{L}{2}\right]$ and setting $v=-u_{x}$ where $u_{x x}=N_{t}$, we formally get two invariant quantities

$$
\begin{aligned}
& \frac{1}{2} \int_{-\frac{L}{2}}^{\frac{L}{2}}\left(F^{2}+G^{2}\right) d x=c_{1} \\
& \frac{1}{2} \int_{-\frac{L}{2}}^{\frac{L}{2}}\left(\left(F_{x}\right)^{2}+\left(G_{x}\right)^{2}+v^{2}+N^{2}+N\left(F^{2}+G^{2}\right)\right) d x=c_{2} .
\end{aligned}
$$

Making an attempt at setting (formally) this system in an hamiltonian form with the notations of Part 2 we set :

$$
X=L^{2} \times L^{2} \times H^{-1} \times H^{-2}
$$

and

$$
Y=H^{1} \times H^{1} \times L^{2} \times H^{-1}
$$




$$
S=\left(\begin{array}{cccc}
-\Delta & 0 & 0 & 0 \\
0 & -\Delta & 0 & 0 \\
0 & 0 & -\Delta & 0 \\
0 & 0 & 0 & -\Delta
\end{array}\right) \text { and } u=(F, G, N, M)
$$

and we get

$$
\frac{1}{2}(S u, u)_{X}=\frac{1}{2} \int_{-\frac{L}{2}}^{\frac{L}{2}}\left(\left(F_{x}\right)^{2}+\left(G_{x}\right)^{2}+v^{2}+N^{2}\right) d x
$$

and thus

$$
g(u)=\frac{1}{2} \int_{-\frac{L}{2}}^{\frac{L}{2}} N\left(F^{2}+G^{2}\right) d x
$$

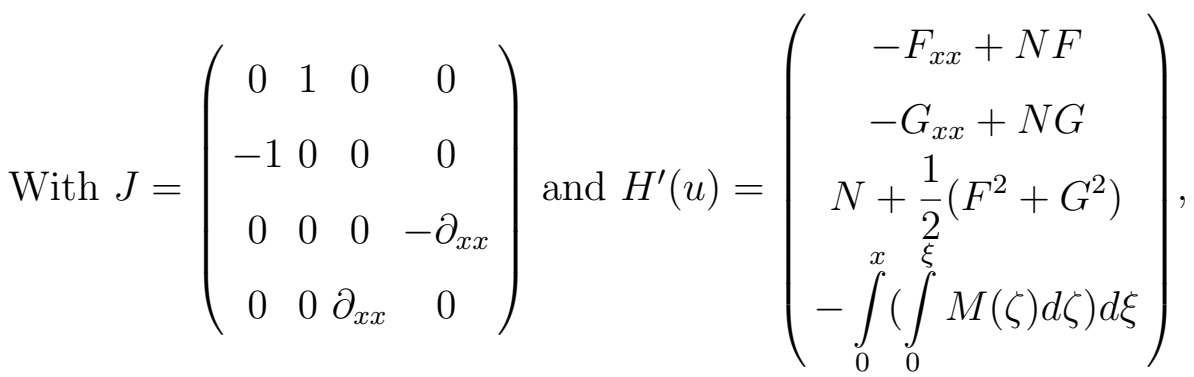

the system (16) is written in an hamiltonian form with the second invariant (18) as hamiltonian.

The hope of getting a result for this continuous equation in such a large space as $X$ is very small, but we can study numerical schemes.

\subsubsection{A space discretization.}

Using notations (4) and (5) for the space derivative as for the previous finite difference schemes, it is possible to discretize (15) in the following way.

$$
\left\{\begin{array}{l}
i \dot{E}_{j}+\delta^{2} E_{j}=N_{j} E_{j}, \\
\ddot{N}_{j}-\delta^{2} N_{j}=\delta^{2}\left(\left|E_{j}\right|^{2}\right),
\end{array}\right.
$$


or setting $\sqrt{2} E_{j}=F_{j}+i G_{j}$ and $M_{j}=\dot{N}_{j}$,

$$
\left\{\begin{array}{l}
\dot{F}_{j}=-\delta^{2} G_{j}+N_{j} G_{j}, \\
\dot{G}_{j}=\delta^{2} F_{j}-N_{j} F_{j}, \\
\dot{N}_{j}=M_{j} \\
\dot{M}_{j}=\delta^{2} N_{j}+\frac{1}{2} \delta^{2}\left(F_{j}^{2}+G_{j}^{2}\right) .
\end{array}\right.
$$

It is possible to find two invariants for this scheme, namely

$$
\begin{aligned}
& \frac{h}{2} \sum_{-K / 2}^{(K / 2)-1}\left\{F_{j}^{2}+G_{j}^{2}\right\}=C_{1}, \\
& \frac{h_{2}}{2} \sum_{-K / 2}^{(K / 2)-1}\left\{\left(\delta F_{j}\right)^{2}+\left(\delta G_{j}\right)^{2}+\left(\delta u_{j}\right)^{2}+N_{j}^{2}+N_{j}\left(F_{j}^{2}+G_{j}^{2}\right)\right\}=C_{1},
\end{aligned}
$$

where $\delta^{2} u_{j}=\dot{N}_{j}$. The setting into an hamiltonian form is made through the following notations : we set $U=\left(\ldots, F_{j}, \ldots, G_{j}, \ldots, N_{j}, \ldots, M_{j}, \ldots\right)$ and

$$
\begin{aligned}
& H(U)=\frac{1}{2}\left\{\left(-\left(D_{K}^{2} F, F\right)-\left(D_{K}^{2} G, G\right)+\left(D_{K}^{2} u, u\right)-(\mathcal{G} M, M)+\right.\right. \\
& \left.+(N, N)+h \sum_{j} N_{j}\left(F_{j}^{2}+G_{j}^{2}\right)\right\} \\
& S=\left(\begin{array}{cccc}
-D_{K}^{2} & 0 & 0 & 0 \\
0 & -D_{K}^{2} & 0 & 0 \\
0 & 0 & I_{K} & 0 \\
0 & 0 & 0 & \mathcal{G}
\end{array}\right)
\end{aligned}
$$

where

$$
\mathcal{G}_{i j}=\left\{\begin{array}{l}
x_{i}\left(1-\frac{x_{j}}{L}\right) \text { si } x_{i} \leq x_{j}, \\
x_{j}\left(1-\frac{x_{i}}{L}\right) \text { si } x_{j} \leq x_{i}
\end{array}\right.
$$

An the hamiltonian form is obtained with : 
$H^{\prime}(U)=\left(\begin{array}{c}-D_{K}^{2} F+N . F \\ -D_{K}^{2} G+N . G \\ N+\frac{1}{2}(F . F+G . G) \\ -\mathcal{G} M\end{array}\right), \quad J=\left(\begin{array}{cccc}0 & I_{K} & 0 & 0 \\ -I_{K} & 0 & 0 & 0 \\ 0 & 0 & 0 & -D_{K}^{2} \\ 0 & 0 & D_{K}^{2} & 0\end{array}\right)$,

where $u . v$ denotes the vector whose components are the $u_{j} v_{j}$.

\subsubsection{A full discretization.}

The following full discretization of the Zakharov equations is due to Glassey $[7]$.

$$
\left\{\begin{array}{l}
i \frac{E_{j}^{n+1}-E_{j}^{n}}{\Delta t}+\frac{1}{2} \delta^{2} E_{j}^{n}+\frac{1}{2} \delta^{2} E_{j}^{n+1}=\frac{1}{4}\left(N_{j}^{n}+N_{j}^{n+1}\right)\left(E_{j}^{n}+E_{j}^{n+1}\right), \\
\frac{N_{j}^{n+1}-2 N_{j}^{n}+N_{j}^{n-1}}{\Delta t^{2}}-\frac{1}{2} \delta^{2} N_{j}^{n+1}-\frac{1}{2} \delta^{2} N_{j}^{n-1}=\delta^{2}\left(\left|E_{j}^{n}\right|^{2}\right) .
\end{array}\right.
$$

As in the nonlinear Schrödinger case, we want to write this scheme in an hamiltonian form. With this aim we set as in the continuous case $\sqrt{2} E_{j}^{n}=$ $F_{j}^{n}+i G_{j}^{n}$ and $M_{j}^{n}=\frac{N_{j}^{n}-N_{j}^{n-1}}{\Delta t}$ which yields the new scheme :

$$
\left\{\begin{array}{l}
\frac{F_{j}^{n+1}-F_{j}^{n}}{\Delta t}=-\frac{1}{2} \delta^{2} G_{j}^{n}-\frac{1}{2} \delta^{2} G_{j}^{n+1}+\frac{1}{4}\left(N_{j}^{n}+N_{j}^{n+1}\right)\left(G_{j}^{n}+G_{j}^{n+1}\right), \\
\frac{G_{j}^{n+1}-G_{j}^{n}}{\Delta t}=\frac{1}{2} \delta^{2} F_{j}^{n}+\frac{1}{2} \delta^{2} F_{j}^{n+1}-\frac{1}{4}\left(N_{j}^{n}+N_{j}^{n+1}\right)\left(F_{j}^{n}+F_{j}^{n+1}\right), \\
\frac{N_{j}^{n+1}-N_{j}^{n}}{\Delta t}=M_{j}^{n+1} \\
\frac{M_{j}^{n+1}-M_{j}^{n}}{\Delta t}=\frac{1}{2} \delta^{2} N_{j}^{n+1}+\frac{1}{2} \delta^{2} N_{j}^{n-1}+\frac{1}{2} \delta^{2}\left(\left(F_{j}^{n}\right)^{2}+\left(G_{j}^{n}\right)^{2}\right)
\end{array}\right.
$$

Glassey proves that for initial data $M_{j}^{1}$ such that $\sum_{j=-K / 2}^{(K / 2)-1} M_{j}^{1}=0$ (zero mean) the numerical scheme is well posed at each step. 
For this scheme we have similar invariants to (17) and (18) :

$$
\begin{aligned}
& \frac{h^{(K / 2)-1}}{\sum_{-K / 2}}\left(\left(F_{j}^{n}\right)^{2}+\left(G_{j}^{n}\right)^{2}\right)=C_{1}, \\
& \frac{h}{2} \sum_{j=-K / 2}^{(K / 2)-1}\left\{\left(\delta F_{j}^{n+1}\right)^{2}+\left(\delta G_{j}^{n+1}\right)^{2}+\left(\delta u_{j}^{n}\right)^{2}+\frac{1}{2}\left(\left(N_{j}^{n}\right)^{2}+\left(N_{j}^{n+1}\right)^{2}\right)\right. \\
& \left.+\frac{1}{2}\left(N_{j}^{n}+N_{j}^{n+1}\right)\left(\left(F_{j}^{n+1}\right)^{2}+\left(G_{j}^{n}\right)^{2}\right)\right\}=C_{2} .
\end{aligned}
$$

where $\delta^{2} u_{j}^{n}=\frac{N_{j}^{n+1}-N_{j}^{n}}{\Delta t}$

\subsection{The Korteweg-de Vries equation.}

We do not know of any finite difference scheme for the Korteweg-de Vries equation with two laws of conservation corresponding to $H_{1}$ and $H_{2}$. In return, problems connected with the nature of the nonlinearity are solved.

As far as we know, the only numerical method which allows a large number of conservation laws is the one developped by Hyman (cf. [9]). This method is based on the work of Lax [12] who shows that a certain class of solutions to the Korteweg-de Vries equation are solutions to a minimization problem. The main idea is to minimize the $N$-th invariant quantity under constraints which are the previous invariants. This is done thanks to an augmented Lagrangian method. This method is not based on an hamiltonian form of the equation. So it is impossible to study it as the restriction of a continuous problem on a finite dimensional phase space. The argument which is used for the different numerical schemes for the nonlinear Schrödinger equation does not apply in the present case. 


\section{Conclusion.}

It seems very difficult to make some great improvements for applying Zhidkov's theoretical frame to partial differential equations. Two facts are responsible for that. First, there are some strong limitations on the nature of both the linear operator and the nonlinearity. Second, in general we need an existence theory in a large space like $L^{2}$ and such results are known for a very limited number of equations especially for periodic boundary conditions. Bourgain has for example also proved some existence results for the Kadomtsev-Petviashvilli II equations in $L^{2}$ for periodic boundary conditions. It seems very difficult to write an hamiltonian numerical scheme for these equations since it is some generalization of the Korteweg-de Vries equation. 


\section{Appendix.}

For the sake of completeness, we cite here the proofs of some results which are only stated in the body of the article. It concerns global existence and uniqueness for the Cauchy problems for the different equations we have studied. We also give useful measure theory results.

\section{A Global existence and uniqueness results.}

We retranscript here Part 4 of Bourgain's article [2] with the adaptation to other nonlinearities. We use the estimates on tori which are explicited in the second part of the same article.

We consider the NLS equation

$$
\begin{aligned}
\Delta u+i \partial_{t} u+f\left(x,|u|^{2}\right) u & =0, \\
u(x, 0) & =\phi(x),
\end{aligned}
$$

where $u$ is periodic in the $x$ variable.

Let us set $w=f\left(x,|u|^{2}\right) u$, the associated integral equation is

$$
u(\cdot, t)=U(t) \phi+i \int_{0}^{t} U(t-\tau) w(\cdot, \tau) d \tau
$$

where $U(t)=e^{i t \Delta}$.

We want to use estimates on tori, hence it is useful to make a time localization. For that aim we introduce a cut-off function $\psi_{1}$ which is equal to 1 on $[-\delta, \delta]$ with a support in $[-2 \delta, 2 \delta]$. It also possible to write the integral equation in the form

$$
u(\cdot, t)=\psi_{1}(t) U(t) \phi+i \psi_{1}(t) \int_{0}^{t} U(t-\tau) w(\cdot, \tau) d \tau .
$$

Using the Fourier transform, we find

$$
u(x, t)=\psi_{1}(t) \sum_{n \in} \hat{\phi}(n) e^{2 \pi i\left(n x+n^{2} t\right)}
$$




$$
+i \psi_{1}(t) \sum_{n \in} e^{2 \pi i\left(n x+n^{2} t\right)} \int_{-\infty}^{+\infty} \frac{e^{2 \pi i\left(\lambda-n^{2}\right) t}-1}{2 \pi i\left(\lambda-n^{2}\right)} \hat{w}(n, \lambda) d \lambda .
$$

We introduce a new cut-off function $\psi_{2}$ which is equal to 1 on $[-B, B]$ and with support in $[-2 B, 2 B]$. Hence we get

$$
\begin{aligned}
& \psi_{1}(t) \int_{-\infty}^{+\infty} \frac{e^{2 \pi i\left(\lambda-n^{2}\right) t}-1}{\lambda-n^{2}} \hat{w}(n, \lambda) d \lambda= \\
& +\sum_{k \geq 1} \frac{(2 \pi i)^{k}}{k !} \psi_{1}(t) t^{k} \int_{-\infty}^{+\infty} \psi_{2}\left(\lambda-n^{2}\right)\left(\lambda-n^{2}\right)^{k-1} \hat{w}(n, \lambda) d \lambda \\
& +\psi_{1}(t) \int_{-\infty}^{+\infty}\left(1-\psi_{2}\right)\left(\lambda-n^{2}\right) \frac{e^{2 \pi i\left(\lambda-n^{2}\right) t}}{\lambda-n^{2}} \hat{w}(n, \lambda) d \lambda \\
& -\psi_{1}(t) \int_{-\infty}^{+\infty}\left(1-\psi_{2}\right)\left(\lambda-n^{2}\right) \frac{\hat{w}(n, \lambda)}{\lambda-n^{2}} d \lambda .
\end{aligned}
$$

It is also necessary to estimate the following terms :

$$
\begin{aligned}
& I=\psi_{1}(t) \sum_{n \in} \hat{\phi}(n) e^{2 \pi i\left(n x+n^{2} t\right)}, \\
& I I=\frac{1}{2 B} \sum_{k \geq 1} \frac{(2 \pi i)^{k}}{k !}(2 B t)^{k} \psi_{1}(t) \\
& \left.\qquad \sum_{n \in}\left[\int_{-\infty}^{+\infty} \psi_{2}\left(\lambda-n^{2}\right)\left(\frac{\lambda-n^{2}}{2 B}\right)^{k-1} \hat{w}(n, \lambda) d \lambda\right] e^{2 \pi i\left(n x+n^{2} t\right)}\right\}, \\
& I I I=\psi_{1}(t) \sum_{n \in} e^{2 \pi i n x} \int_{-\infty}^{+\infty} \frac{\left(1-\psi_{2}\right)\left(\lambda-n^{2}\right)}{\lambda-n^{2}} e^{2 \pi i \lambda t} \hat{w}(n, \lambda) d \lambda, \quad \\
& I V=-\psi_{1}(t) \sum_{n \in} e^{2 \pi i\left(n x+n^{2} t\right)} \int_{-\infty}^{+\infty} \frac{\left(1-\psi_{2}\right)\left(\lambda-n^{2}\right)}{\lambda-n^{2}} e^{2 \pi i \lambda t} \hat{w}(n, \lambda) d \lambda .
\end{aligned}
$$

In the same way as in [2], we get

$$
\begin{aligned}
& \|I\|_{L^{4}(d x d t)} \leq c\|\phi\|_{2}, \\
& \|I I\|_{L^{4}(d x d t)} \leq c \delta B\|w\|_{L^{4 / 3}(d x d t)}, \\
& \|I I I\|_{L^{4}(d x d t)} \leq C B^{-1 / 4}\|w\|_{L^{4 / 3}(d x d t)},
\end{aligned}
$$




$$
\|I V\|_{L^{4}(d x d t)} \leq C B^{-1 / 4}\|w\|_{L^{4 / 3}(d x d t)} .
$$

With the aim to have the same sort of estimates than Bourgain we impose for example that

$$
\begin{aligned}
& \|w\|_{4 / 3} \leq C \sum_{\beta}\|u\|_{4}^{\beta}, \\
& \left\|w_{1}-w_{2}\right\|_{4 / 3} \leq C \sum_{\gamma}\left(\left\|u_{1}\right\|_{4}+\left\|u_{2}\right\|_{4}\right)^{\gamma}\left\|u_{1}-u_{2}\right\|_{4},
\end{aligned}
$$

where $w_{i}=f\left(x,\left\|u_{i}\right\|^{2}\right) u_{i}, i=1,2$, the sums over $\beta$ or $\gamma$ containing a finite number of terms and $1 \leq \beta, 0 \leq \gamma$. Let us set

$$
\begin{aligned}
T u(x, t) & =\psi_{1}(t) \sum_{n \in} \hat{\phi}(n) e^{2 \pi i\left(n x+n^{2} t\right)} \\
& +i \psi_{1}(t) \sum_{n \in} e^{2 \pi i\left(n x+n^{2} t\right)} \int_{-\infty}^{+\infty} \frac{e^{2 \pi i\left(\lambda-n^{2}\right) t}-1}{2 \pi i\left(\lambda-n^{2}\right)} \hat{w}(n, \lambda) d \lambda .
\end{aligned}
$$

The proof of the global existence in $L^{2}$ is carried out as follows :

- there exists a constant $M$ such that $T$ maps $B(0, M)$ in itself ;

- on this ball $T$ is a contraction (for these two points we have to impose conditions on $\delta$ and $B$ ) ;

- thanks to a fixed point argument the problem is locally well posed in $L^{2}$;

- according to the conservation of the $L^{2}$ norm for this equation, the solution is global in time.

Les us check the first two points :

$$
\|T u\|_{4} \leq c_{1}\left\{\|\phi\|_{2}+\delta B\left(\sum_{\beta}\|u\|_{4}^{\beta}\right)+B^{-1 / 4}\left(\sum_{\beta}\|u\|_{4}^{\beta}\right)\right\},
$$

which may be brought to be lower than $M$, for a sufficiently large $M$, since it is possible to fix $\delta B$ and $B^{-1 / 4}$ arbitrarily small.

Hence $\|u\|_{4} \leq M$ implies that $\|T u\|_{4} \leq M$.

$$
\begin{aligned}
& \left\|T u_{1}-T u_{2}\right\|_{4} \leq c_{1}\left(\delta B+B^{-1 / 4}\right) \sum_{\gamma}\left(\left\|u_{1}\right\|_{4}+\left\|u_{2}\right\|_{4}\right)^{\gamma}\left\|u_{1}-u_{2}\right\|_{4}, \\
& \leq 2 c_{1}\left(\delta B+B^{-1 / 4}\right) \sum_{\gamma}(M)^{\gamma}\left\|u_{1}-u_{2}\right\|_{4} .
\end{aligned}
$$

The quantity $2 c_{1}\left(\delta B+B^{-1 / 4}\right) \sum_{\gamma}(M)^{\gamma}$ may be brought to be lower than $\frac{1}{2}$ up 
to a new decreasing of $\delta B$ and $B^{-1 / 4}$ which is compatible with the former one. We now give a few fields of application for this result.

The case which is studied by Bourgain is $w=|u|^{\alpha} u$. He shows that the two hypotheses on $w$ are verified in the case when $0<\alpha \leq 2$.

The two nonlinearities Zhidkov proposes are $w=\frac{u|u|^{2}}{1+|u|^{2}}$ and $w=1-e^{-\alpha u}$. In the frame we adopt here, we want that

$$
\begin{aligned}
& \left\|f\left(x,|u|^{2}\right) u\right\|_{4 / 3} \leq C \sum_{\beta}\|u\|_{4}^{\beta}, \\
& \left\|f\left(x,|u|^{2}\right) u-f\left(x,|v|^{2}\right) v\right\|_{4 / 3} \leq C \sum_{\gamma}\left(\|u\|_{4}+\|v\|_{4}\right)^{\gamma}\|u-v\|_{4} .
\end{aligned}
$$

The first estimate is true if

$$
\left\|f\left(x,|u|^{2}\right)\right\|_{2} \leq C \sum_{\gamma}\|u\|_{4}^{\gamma} .
$$

The second may be fulfilled under one of the two following conditions :

$$
\begin{aligned}
& \sup _{u \in B(0, M)}\left\|\nabla_{s} f\left(x,|u|^{2}\right) u\right\|_{2} \leq C \sum_{\gamma} \sup _{u \in B(0, M)}\|u\|_{4}^{\gamma} \text { when } u \in B(0, M), \\
& \text { or }\left\|\nabla_{s} f\left(x,|u|^{2}\right)\right\|_{4} \leq C \sum_{\eta} \sup _{u \in B(0, M)}\|u\|_{4}^{\eta} \text { when } u \in B(0, M),
\end{aligned}
$$

the sum over $\eta$ containing a finite number of terms and $-1 \leq \eta$. 


\section{B Gaussian measures on a Hilbert space.}

The following ingredients are contained in Dalecky and Fomin's book [5]. This appendix is by no means meant to be an abstract about Gaussian measures in Hilbert spaces, we only give some hints to make clear the proof of Lemma 3 .

Let us begin with a theorem which gives a condition for a premeasure to be $\sigma$-additive.

Let $\nu$ be a premeasure on an algebra $\mathcal{U}$ of subsets of a set $X$. We say that a class $\mathcal{K} \subset \mathcal{U}$ approximates $\nu$ from below if for each $A \in \mathcal{U}$ and each $\varepsilon>0$, there exists a $K \in \mathcal{K}$ such that $K \subset A$ and $|\nu|(A \backslash K)<\varepsilon$.

According to this definition, we may state :

Proposition B.1 Let $\nu$ be a premeasure on an algebra $\mathcal{U}$ of subsets of a topological space $X$ and let $\mathcal{F} \subset \mathcal{U}$ be a class of closed subsets of $X$, which is closed under intersection and approximates $\nu$ from below.

If for every $\varepsilon>0$, there exists a compact set $K_{\varepsilon}$ such that for all $F \in \mathcal{F}$,

$$
F \cap K_{\varepsilon}=\emptyset \Rightarrow|\nu|(F)<\varepsilon,
$$

then $\nu$ is $\sigma$-additive, i.e. it is a measure.

On a Hilbert space, we can define particular measures called Gaussian cylindrical measures. The construction is carried out as follows.

Let $\nu$ be a measure on ${ }^{n}$, its characteristic functional $\chi_{\nu}$ is given by the formula

$$
\chi_{\nu}(\xi)=\int_{n} e^{i \xi \cdot x} \rho(x) d x
$$

where $\rho$ is the density of $\nu$ with respect to Lebesgue's measure $d x$. The measure $\nu$ is said to be Gaussian if its characteristic functional may be expressed in the form

$$
\chi_{\nu}(y)=\exp \left\{-\frac{1}{2}(B y, y)+i(\alpha, y)\right\}
$$

where $B$ is a positive operator. It is said to be centered if $\alpha$ is zero.

In a Hilbert space $X$ we call cylindrical a set in the form

$$
\mathbf{C}=\{x \in X ; P x \in \mathcal{M}\},
$$

where $P$ is a projection onto a subset of a finite dimension of $X$, and $\mathcal{M}$ is a 
Borel set of this subspace called the base of $\mathbf{C}$.

We define a cylindrical measure $\nu$ over $X$ by the measure of the cylindrical sets of $X$. The measure of a cylindrical set $\mathbf{C}$ is chosen to be equal to the measure of the base $\mathcal{M}$ of $\mathbf{C}$ in $P X$.

Such a measure is said to be a centered Gaussian measure if each projection onto a finite dimensional space is a centered Gaussian measure. Hence its characteristic functional is in the form

$$
\chi_{\nu}(y)=e^{-b(y, y) / 2}
$$

where $b$ is a nonnegative bilinear form which is continuous on every finite dimensional subset and is called the correlation of $\nu$. If $b(y, y)$ may be written in the form $(B y, y), B$ is called the correlation operator of $\nu$. We will denote by $\nu_{B}$ the centered Gaussian cylindrical measure with correlation operator $B$. Now we have the lemma

Lemma B.2 Let $A$ be a positive operator then

$$
\begin{aligned}
& \nu_{B}\left\{x:(A x, x)_{X} \geq 1\right\} \leq \operatorname{Tr} A B, \\
& \nu_{B}\{x:|(A x, x)-\operatorname{Tr} A B| \leq c \sqrt{\operatorname{Tr} A B}\} \geq 1-\frac{2}{c^{2}}\|A B\|_{X} .
\end{aligned}
$$

It is now possible to prove lemma 3 which gives a necessary and sufficient condition of $\sigma$-additivity for a centered Gaussian cylindrical measure.

Lemma 3. A centered Gaussian cylindrical measure $\nu$ on $X$ is $\sigma$-additive if and only if its correlation operator is in the form $b\left(y_{1}, y_{2}\right)=\left(B y_{1}, y_{2}\right)$, where $B$ is a nuclear positive operator $X$.

\section{Sufficient condition :}

If $\nu$ is $\sigma$-additive then its characteristic functional and its correlation are continuous on $X$, hence $b(y, y)=(B y, y)$ with $B \in \mathcal{L}(X)$. Let us show by contradiction that $B$ is nuclear. If it is not the case, we may find a projection onto a finite dimensional subspace such that its trace $\operatorname{Tr} P B P=R$ is arbitrarily large. Then we set

$$
\mathbf{C}=\left\{x \in X:\left|\|P x\|_{X}^{2}-R\right|<\alpha \sqrt{R}\right\} .
$$

The intersection of this cylinder with the ball of center 0 and radius $R-\alpha \sqrt{R}$ is empty.

According to lemma 1.1, we have

$$
\nu(\mathbf{C}) \geq 1-\frac{2\|b\|}{\alpha^{2}}=\frac{1}{2} \text { if we take } \alpha=2 \sqrt{\|B\|} .
$$


We may consider balls of $X$ centered in 0 , with an arbitrarily large radius and with a measure which is lower than $\frac{1}{2}$. This is impossible since $\nu(X)=1$.

Necessary condition :

Let $\mathbf{C}$ be a cylinder which does not intersect the ball in $X$ with center 0 and radius $R$. Its base $\mathcal{M}$ also does not intersect the ball of center 0 and radius $R$ in $P X$. According to lemma 1.1, we get

$$
\nu(\mathbf{C}) \leq \frac{\operatorname{Tr} P B P}{R^{2}} \leq \frac{\operatorname{Tr} B}{R^{2}}
$$

which is finite since we assume that $B$ is nuclear. We use proposition B.1 with $\mathcal{U}$ equal to the Borel $\sigma$-algebra of $X$ and $\mathcal{F}$ to the set of cylinders in $X$.

For all $\varepsilon>0$, there exists $K_{\varepsilon}=B(0, R)$ with $\varepsilon=\frac{\operatorname{Tr} B}{R^{2}}$ such that, for all $F \in \mathcal{F}$ such that $F \cap B(0, R)=\emptyset$, then $\nu(F) \leq \varepsilon$. 


\section{References}

[1] V.I. Arnold Mathematical Methods of Classical Mechanics. Springer, New York, 1978

[2] J. Bourgain Fourier transform restriction phenomena for certain lattice subsets and applications to non-linear evolution equations. Part I : Schrödinger Equations. GAFA, 3, 107-156 (1993)

[3] J. Bourgain Fourier transform restriction phenomena for certain lattice subsets and applications to non-linear evolution equations. Part II : The KdVEquation. GAFA, 3, 209-262 (1993)

[4] J. Bourgain Periodic Nonlinear Schrödinger Equation and Invariant Measure. Preprint, IHES

[5] Yu. L. Dalecky, S.V. Fomin Measures and Differential Equations in InfiniteDimensional Space. Kluwer Academic Publishers, 1991

[6] L. Friedlander An invariant measure for the equation $u_{t t}-u_{x x}+u^{3}=0$. Commun. Math. Phys., 98, 1-16 (1985)

[7] R.T. Glassey Convergence of an Energy-preserving scheme for the Zakharov equations in one space dimension. Prepr. Indiana-Univ. Bloomington

[8] B.M. Herbst, M.J. Ablowitz On the numerical chaos in the nonlinear Schrödinger Equation.

[9] J.M. Hyman Time Evolution of almost periodic solutions of the KdV equation. Rocky Mountain J. of Math., 8, 95-104 (1978)

[10] E. Infeld Quantitive theory of the Fermi-Pasta-Ulam Recurrence in the Nonlinear Schrödinger Equation. Phys. Rev. Letters, 47, 717-718 (1981)

[11] P.A.E.M. Janssen Modulational instability and the Fermi-Pasta-Ulam recurrence. Phys. Fluids, 24, 23-26 (1981)

[12] P.D. Lax Periodic Solutions of the KdV Equation. Commun. in Pure and Appl. Math., 28, 141-188 (1975)

[13] P.D. Lax Almost Periodic Behavior of Nonlinear Waves. Advances in Math., 16, 368-379 (1975)

[14] J.L. Lebowitz, H.A. Rose, E.R. Speer Statistical Mechanics of the Nonlinear Schrödinger Equation. J. of Stat. Phys., 50, 657-687 (1988)

[15] H.P. McKean, E. Trubowitz Hill's operator and hyperelliptic function theory in the presence of infinitely many branch points. Comm. Pure Appl. Math. 29, 143-226 (1976)

[16] D.U. Martin, H.C. Yuen Quasi-recurring energy leakage in the two-spacedimensional nonlinear Schrödinger Equation. Phys. Fluids, 23, 881-883 (1980) 
[17] P.J. Olver Applications of Lie Groups to Differential Equations. Springer Verlag, 1986

[18] K. Rachid Simulation numérique des systèmes de Davey-Stewartson. Thèse de l'Université Paris-Sud, 1993

[19] Y. Tsutsumi $L_{2}$-solutions for non linear Schrödinger equations and nonlinear Groups. Funkcialaj Ekvac., 30, 115-125 (1987)

[20] J.A.C. Weideman, B.M. Herbst Recurrence in semidiscrete approximations of the nonlinear Schrödinger Equation. SIAM J. on Scient. and Stat. Comp., 8, 98-1004 (1987)

[21] H.C. Yuen, W.E. Ferguson Jr. Relationship between Benjamin-Feir instability and recurrence in the nonlinear Schrödinger equation. Phys. Fluids, 21, 1275$1278(1978)$

[22] H.C. Yuen, W.E. Ferguson Jr. Fermi-Pasta-Ulam recurrence in the twospace dimensional nonlinear Schrödinger equation. Phys. Fluids, 21, 2116-2118 (1978)

[23] H.C. Yuen, B.M. Lake, W.E. Ferguson The significance of Nonlinearity in the Natural Science. Plenum, New York, 67ff. (1977)

[24] V.E. Zakharov Collapse of Langmuir Waves. Soviet Physics JETP., 35, 908-914 (1972)

[25] P.E. Zhidkov On the invariant measure for the nonlinear Schrödinger equation. JINR, E5-91-174 (1991)

[26] P.E. Zhidkov On an invariant measure for a nonlinear Schrödinger equation. Sov. Math. Dokl., 43 (1991)

[27] P.E. Zhidkov A remark on the invariant measure for the nonlinear Schrödinger equation. JINR, E5-92-23 (1992)

[28] P.E. Zhidkov An invariant measure for a nonlinear wave equation. JINR, E592-305 (1992)

[29] P.E. Zhidkov On invariant measures for some infinite-dimensional dynamical systems. JINR, E5-92-395 (1992) 